\title{
Applicability of an orthogonal cutting slip-line field model for the microscale
}

\author{
Lara Rebaioli $^{1}$, Gabriele Biella ${ }^{2}$, Massimiliano Annoni ${ }^{3}$, J. Rhett Mayor ${ }^{4}$, Quirico \\ Semeraro $^{5}$
}

${ }^{1}$ Dipartimento di Meccanica, Politecnico di Milano, Italy; lara.rebaioli@polimi.it, +39 0223998592

${ }^{2}$ Dipartimento di Meccanica, Politecnico di Milano, Italy

${ }^{3}$ Dipartimento di Meccanica, Politecnico di Milano, Italy

${ }^{4}$ The George W. Woodruff School of Mechanical Engineering, Georgia Institute of Technology, Atlanta, USA

${ }^{5}$ Dipartimento di Meccanica, Politecnico di Milano, Italy

\begin{abstract}
Mechanical micromachining is a very flexible and widely exploited process, but its knowledge should still be improved since several incompletely explained phenomena play a role on the microscale chip removal (e.g. "minimum chip thickness effect", microstructure influence on cutting forces, stable built-up edge, etc.). Several models have been developed to describe the machining process, but only some of them take into account a rounded-edge tool, which is a typical condition in micromachining. Among these models, the slip-line field model developed by Waldorf for the macroscale allows to separately evaluate shearing and ploughing force components in orthogonal cutting conditions, therefore it is suitable to predict cutting forces when a large ploughing action occurs, as in micromachining. The present study aims at demonstrating how this model is suitable also for micromachining conditions. In order to achieve this goal, a clear, modular and repeatable procedure has been developed for objectively validating its cutting and feed force prediction performance at low uncut chip thickness (less than $50 \mu \mathrm{m}$ ) and relatively higher cutting edge radius. The proposed procedure makes the model generally applicable after a suitable and
\end{abstract}


non-extensive calibration campaign. The present paper shows how calibration experiments can be selected among the available database of cutting trials basing on the model force prediction capability. Final validation experiments have been used to show how the model is robust to a cutting speed variation even if the cutting speed is not among the model quantities. A suitable set-up, especially designed for microturning conditions, has been used in this research to measure forces and chip thickness. Tests have been carried out on 6082-T6 Aluminum alloy with different cutting speeds and different ratios between uncut chip thickness and cutting edge radius.

KEYWORDS: micromachining, chip formation, stable built-up edge, cutting forces, orthogonal cutting

\section{NOMENCLATURE}

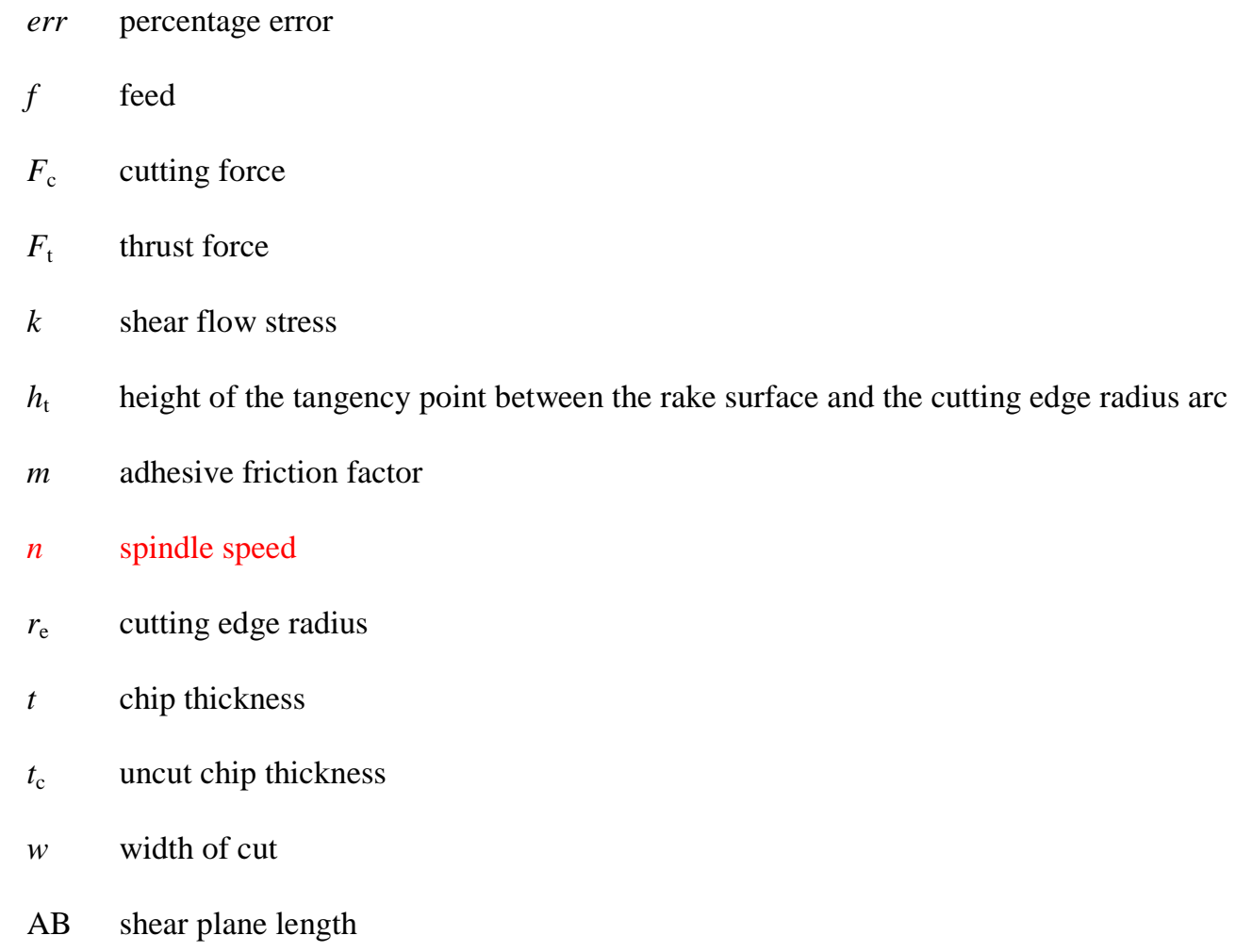




$$
\begin{array}{ll}
\text { CA } & \text { ploughing contact length } \\
R & \text { fan field radius } \\
V_{\mathrm{c}} & \text { cutting speed } \\
V_{\mathrm{f}} & \text { feed rate } \\
\alpha & \text { rake angle } \\
\alpha_{\mathrm{eff}} & \text { effective rake angle } \\
\gamma & \text { slip-line field angle } \\
\eta & \text { slip-line field angle } \\
\theta & \text { slip-line field angle } \\
\rho & \text { prow angle } \\
\phi & \text { shear angle }
\end{array}
$$

\section{Subscripts}

cal calibration

meas measured

pred predicted

- "W" for the Waldorf model or "WE" for the Waldorf model considering the effective rake angle

- "cc" for cutting or " $\mathrm{t}$ " for thrust

\section{INTRODUCTION}

Among the different available processes, micro mechanical machining is one of the most flexible and widely exploited ones, even if its characterizing phenomena are still not well understood and controlled; basic knowledge about the physical phenomena [1-2] should still be improved and, in particular, the following scale-effects need to be further studied: 
- if critical tool geometrical features, as the edge radius, assume the same magnitude order of the chip thickness, the tool effective rake angle gets highly negative, ploughing forces become more relevant than shearing forces and the cutting specific energy gets higher [3-4];

- chip removal becomes discontinuous since a minimum uncut chip thickness exists under which no material can be removed from the workpiece ("minimum chip thickness effect") [3-6];

- the workpiece material microstructure plays an important role on cutting forces, since the tool geometrical features tend to be comparable in size to the target material grains, and make them vary with the grain orientation [7-8];

- a stable built-up edge, named "dead metal cap", is claimed by some researchers to be always present when machining with an uncut chip thickness lower than the tool cutting edge radius [9-11].

\subsection{State of the Art}

Over the years, the chip removal process has been described by several models; Lee and Shaffer [12] firstly presented a model based on the so-called "slip-line field" theory, potentially able to predict also the ploughing effects. This is the main reason why slip-line field theory is the focus of the present paper.

The existing slip-line field models consider different tool shapes, which are mainly the following:

- sharp ([13-20]);

- rounded ([9-10, 21-29]);

- chamfered ([30-31]);

- worn $([20,32])$.

The typical microscale conditions can be better represented by taking into account a rounded tool shape. In fact, the cutting edge radius is always significant at this scale, due to current tool fabrication process capability that prevents it to be lower than typical chip thickness at the microscale. In recent years, many slip-line field models have been specifically developed for the microscale [9-10, 21-29] and all of them consider a rounded tool edge. 
The above cited models can be divided in two groups taking into account the applied modeling approach:

- the assumption of a stable built-up edge (according to experimental findings of [11]) was made by Waldorf et al. [9-10], Liu et al. [23-25], Karpat et al. [26], Yoon et al. [27] and Ozturk et al. [29];

- the assumption of a separation point on the tool edge diverting the material flow was made by Fang et al. [21-22] and Jin et al. [28].

Among the first model group, the study by Waldorf et al. [9-10] presented a slip-line field model consisting of three shear zones, which is able to predict also the ploughing force components. The authors made the assumptions of constant friction factor and rigid, perfectly plastic material (i.e. the shear flow stress only depends on the machined material but not on strain, strain rate or temperature). The authors validated their model by performing orthogonal turning tests on Al 6061-T6 [9].

In their study, Liu et al. [23-25] proposed a slip-line field similar to the Fang's one [21-22] but considering only slip-lines necessary for cutting force calculations. Moreover, the authors took into account the dead metal cap presence and introduced the effective rake angle in the model as a function of the uncut chip thickness [33].

In their paper, Karpat et al. [26] proposed a model derived from Waldorf's one in which the friction factor was calculated basing on cutting forces and acquired chip geometry. The authors performed a finite elements analysis, applying the Johnson-Cook model, to evaluate the shear flow stress. They also carried out orthogonal turning tests on AISI 4340 steel with an uncut chip thickness of 100, 125 and $150 \mu \mathrm{m}$ (corresponding to a normalized uncut chip thickness $t_{\mathrm{c}} / r_{\mathrm{e}}$ between 2.5 and 3.75 , since the used cutting edge radii were 40 and $50 \mu \mathrm{m}$ ) and a cutting speed of 125 and $175 \mathrm{~m} / \mathrm{min}$.

Yoon et al. [27] designed a slip-line field model including the minimum chip thickness effect and the effective rake angle; they also supposed the friction factor to be constant. The model was validated by means of microturning tests on 260 brass alloy $(\mathrm{CuZn30)}$ with an uncut chip thickness ranging from 1 to $13 \mu \mathrm{m}$ and a cutting edge radius of $5 \mu \mathrm{m}$. 
In their study, Ozturk et al. [29] presented a slip-line field composed by eight shear regions and including a dead metal cap, under the assumptions of constant friction factor and rigid, perfectly plastic material. In order to validate the model, some orthogonal cutting experiments were performed on 260 brass alloy $(\mathrm{CuZn30})$ using tools with a cutting edge radius of 50, 100 and $150 \mu \mathrm{m}$; the uncut chip thickness was equal to 50,100 and $150 \mu \mathrm{m}$ (hence the normalized uncut chip thickness $t_{\mathrm{c}} / r_{\mathrm{e}}$ ranged from 0.33 to 3 ) and the selected cutting speeds were $0.25,0.50$ and $0.75 \mathrm{~m} / \mathrm{min}$ in order to avoid thermal effects. This paper also shows some chip micrographs obtained by a quick stop device successfully operating up to a maximum cutting speed of $17.5 \mathrm{~m} / \mathrm{min}$.

Among the second model group, the model by Fang et al. [21-22] is composed by twenty seven slip-line subregions and allows to estimate also the ploughing force components. The mathematical formulation of this model is based on Dewhurst and Collins matrix technique [15, 34] and carries out a non-unique solution. The predicted forces were compared with experimental observations coming from past works by other authors.

Jin et al. [28] designed a model which divides the material deformation region into three main zones; they applied the Johnson-Cook constitutive model to obtain the shear flow stress and the hydrostatic pressure as functions of strain, strain-rate and temperature. The authors validated their model by carrying out turning experiments on 260 brass alloy (CuZn30) using a tool with a $20 \mu \mathrm{m}$ cutting edge radius and a cutting speed between 150 and $350 \mathrm{~m} / \mathrm{min}$. The selected uncut chip thickness values were between 15 and $80 \mu \mathrm{m}$, in order to obtain a ratio with the cutting edge radius ranging from 0.75 to 4 .

These orthogonal cutting models are useful to describe the chip formation process and, hence, to improve the knowledge on the machining process. Orthogonal cutting models based on the slip-line theory can also be used for other purposes, e.g. some researchers developed milling models based on an orthogonal cutting slip-line field that is linked to the milling process by a proper chip thickness analytical function. This 
approach was used in the studies of Jun et al. [23-24] and Altintas et al. [35], which are respectively based on the slip-line field models by Waldorf et al. [9] and Jin et al. [28].

\subsection{Objectives}

As discussed for the state of the art, an experimental validation of models presented in literature has just been made in some of the analyzed papers [9-10, 26-29]. The present paper focuses on the Waldorf's model [9] with the purpose to validate it in the micromachining field.

This model has been selected since it is simple, it has only a few parameters to experimentally calibrate (no FEM analysis is needed) and, at the same time, it allows to evaluate cutting forces when a strong ploughing occurs (i.e. when the uncut chip thickness is lower than the tool edge radius), i.e. in the typical micromachining conditions. Moreover, Waldorf et al. [10] showed how models including a stable built-up edge have a better prediction performance than models assuming the material separation at a certain point on the tool cutting edge.

After taking the Waldorf model [9-10] as reference, the present study has developed a repeatable procedure to calibrate it for a specific target material by means of a suitable restricted set of experiments [36]. Such a calibration procedure allows to make the model a useful tool to predict the cutting and thrust forces in different conditions of feed and cutting speed that are significant for the micro field and outside the calibration window. This study demonstrates this result for both the original Waldorf model and for a modified version considering the effective rake angle [36]. An objective comparison between performances of the two versions is also presented to confirm the usefulness of the effective rake angle introduction [36]. Introducing the effective rake angle allows to account for typical low chip thickness effects on microcutting, which make the rake angle strongly negative, the ploughing forces sometimes more relevant than shearing forces and the specific cutting energy extremely high. Moreover, the effective rake angle introduction allows modeling a dead metal cap size changing with the uncut chip thickness. Finally, a model validation has been presented where cutting speed has been varied comparing to calibration 
experiments in order to show how the model is robust to a cutting speed variation.

\section{THE WALDORF'S SLIP-LINE FIELD MODEL}

Waldorf et al. [9-10] developed a slip-line field model for the orthogonal cutting condition that is able to predict the cutting and thrust force components, also considering their shear and ploughing portion. Fig. 1 depicts this field that partly derives from previous slip-line fields in literature. In particular, it resembles the model developed by Shi and Ramalingam for cutting with flank wear [32], that, in turn, has been developed basing on a slip-line solution for orthogonal cutting proposed by Kudo [14].

The slip-line field proposed by Waldorf consists of three regions of rigid material motion:

- the first region (I in Fig. 1) is a pre-flow zone where a material raising takes place due to compressive stresses; the prow angle ( $\rho$ in Fig. 1) accounts for this effect;

- the second region (II in Fig. 1) corresponds to the traditional "primary shear zone" that is defined by the shear angle ( $\phi$ in Fig. 1);

- the third region (III in Fig. 1) is the "tertiary shear zone", which is close to the dead metal cap. Its dimensions depend on the friction conditions at the tool-chip interface.

The "secondary shear zone", where the tool rake face gets in contact with the chip, is not considered in the Waldorf model. 


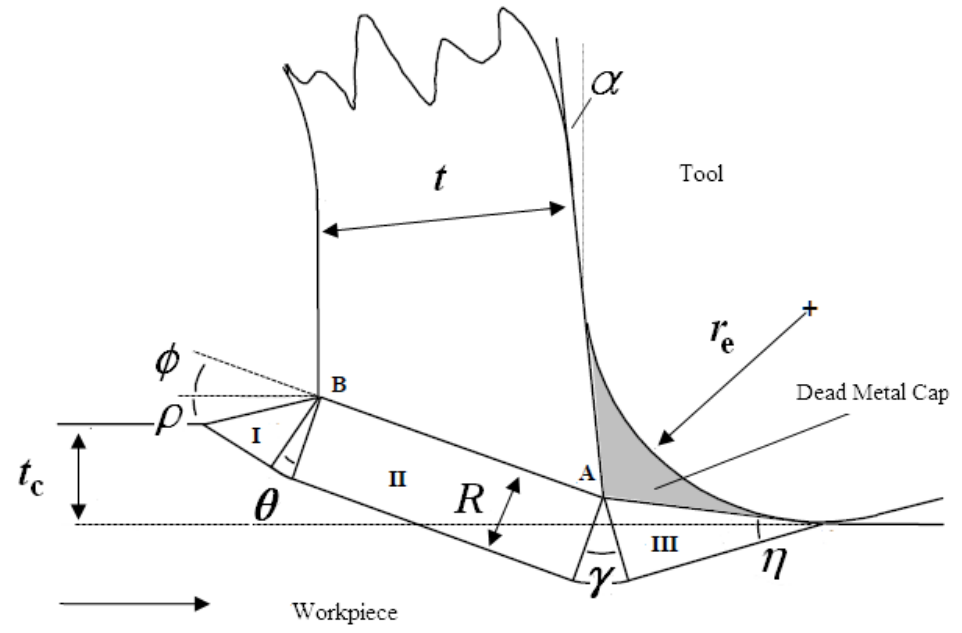

Fig. 1: Slip-line field proposed by Waldorf (adapted from [9]).

Some of the model parameters are immediately available once defined the experimental conditions, i.e. they are defined outside the model and do not need to be calibrated:

- $m$ (adhesive friction factor, i.e. the ratio between shear and flow stress) $=0.99$, as stated by Waldorf [9]; - $r_{\mathrm{e}}$ (cutting edge radius) $=35 \mu \mathrm{m}$ and $\alpha$ (rake angle $)=16^{\circ}$, obtained by measurements made on the insert used in this paper (see Section 3.2) by an Alicona Infinite Focus optical 3D measuring system;

- $t_{\mathrm{c}}$ (uncut chip thickness) equal to the feed $f$, since orthogonal cutting hypotheses are satisfied in microturning experiments (see Section 3.2);

- $w$ (width of cut $)=0.73 \mathrm{~mm}$, obtained as the mean of measurements made on the workpieces by a Zeiss Prismo 5 VAST MPS HTG Coordinate Measuring Machine.

Only three model input parameters $(\phi, k, \rho)$ have to be calibrated: this fact confirms the model simplicity. Section 4 presents equations and techniques useful to this purpose. 


\section{CALIBRATION AND VALIDATION EXPERIMENTAL DATABASE}

\subsection{Experimental Design}

A factorial experiment (whose factors are summarized in Tab. 1) has been designed in order to build an experimental database useful to calibrate and validate the Waldorf model, in case of both the original model version [9-10] and the Waldorf model considering the effective rake angle.

Tab.1: Experimental design factors.

\begin{tabular}{|l|l|l|}
\hline Factor & Symbol & Levels \\
\hline \hline Cutting speed & $V_{\mathrm{c}}$ & $150,200,250 \mathrm{~m} / \mathrm{min}$ \\
\hline Normalized uncut chip thickness & $t_{\mathrm{c}} / r_{\mathrm{e}}$ & $0.4,0.6,0.8,1$ \\
\hline
\end{tabular}

The cutting speed factor varies on three levels: 150, 200 and $250 \mathrm{~m} / \mathrm{min}$. Such values have been selected in a range where cutting takes place satisfactorily (no vibrations, no burrs, good surface finish) according to preliminary tests. The considered normalized uncut chip thickness levels are four: 0.4, 0.6, 0.8 and 1 , corresponding respectively to an uncut chip thickness of $14,21,28$ and $35 \mu \mathrm{m}$. These values have been selected in order to ensure a regular chip formation by staying immediately above the commonly accepted value of minimum uncut chip thickness, equal to $0.3 \div 0.4 r_{\mathrm{e}}[4]$.

Four replicates have been carried out for each factors combination, hence the experimental design (Fig. 2) consists of 48 runs, which have been completely randomized. 


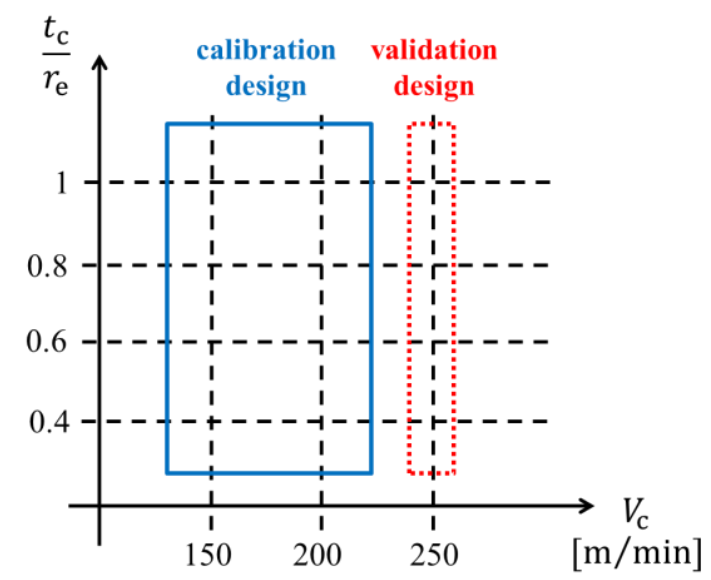

Fig. 2: Experimental database.

The whole experimental database has been divided in two parts: the calibration design (blue solid box in Fig. 2) has provided the experimental condition sets for the model calibration (see Section 5), while the validation design (red dotted box in Fig. 2) has been used to validate the model prediction performance (see Section 6).

The cutting speed is not a model input parameter, however, it is known that the cutting speed affects the material properties (e.g. a cutting speed increase causes a softening effect), hence the selected model has been validated also for a different cutting speed level in order to prove its robustness. It seems reasonable not to exceed with the cutting speed values since they are typically low in micromachining.

\subsection{Experiment description}

Microturning operations have been performed by means of a suitable set-up designed to be used on the Kern EVO ultra precision 5-axis machining center available at the "MI_crolab" of Dipartimento di Meccanica of Politecnico di Milano (nominal positioning tolerance $= \pm 1 \mu \mathrm{m}$, precision on the workpiece $=$ $\pm 2 \mu \mathrm{m}$ ). A Kistler 9257BA triaxial piezoelectric load cell (D in Fig. 3) has been employed to measure 
cutting forces during each test.

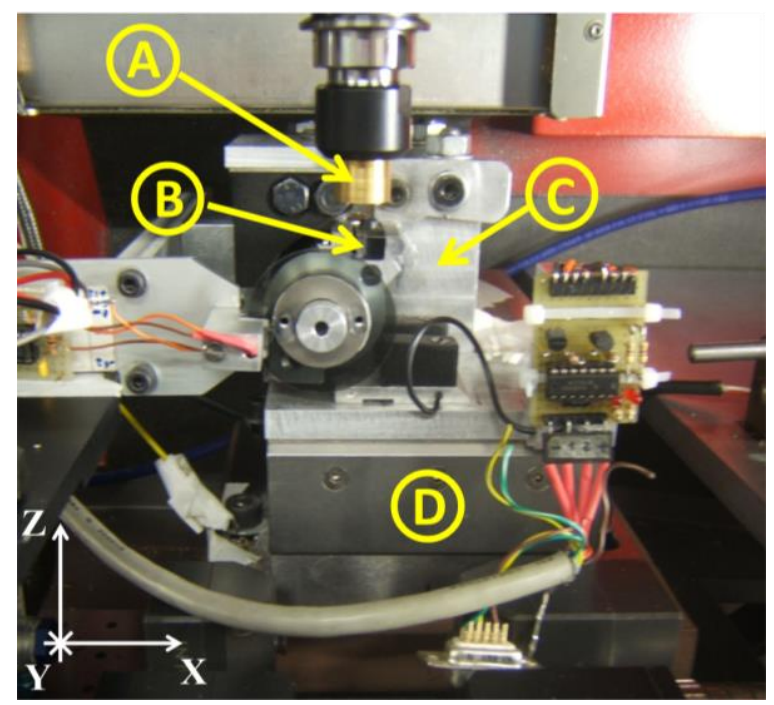

Fig. 3: Microturning set-up [A = workpiece, $B=$ tool, $C=$ tool holder, $D=$ load cell].

The orthogonal cutting condition (Fig. 4) has been obtained by turning a thin-walled tubular workpiece $($ diameter $=15 \mathrm{~mm}$, nominal wall thickness $=0.7 \mathrm{~mm})$, mounted on the machine spindle $(\mathrm{A}$ in Fig. 3$)$ and moving at the cutting speed $V_{\text {c. }}$ The turning tool carrying out the operations (B in Fig. 3) has been fixed to the machine table by a proper tool holder ( $\mathrm{C}$ in Fig. 3). Thanks to this set-up, the orthogonal cutting condition exists since the following hypotheses are satisfied: the cutting edge is perpendicular to the cutting speed and wider than the width of cut $w$ (i.e. the chip has no constraints at its sides), the cutting speed has acceptable variations along the cutting edge and the uncut chip thickness $t_{\mathrm{c}}$ is much lower than the width of cut $w$. Moreover, the uncut chip thickness $t_{\mathrm{c}}$ can be considered equal to the actual feed $f$ since the feed rate is negligible comparing to the cutting speed. 

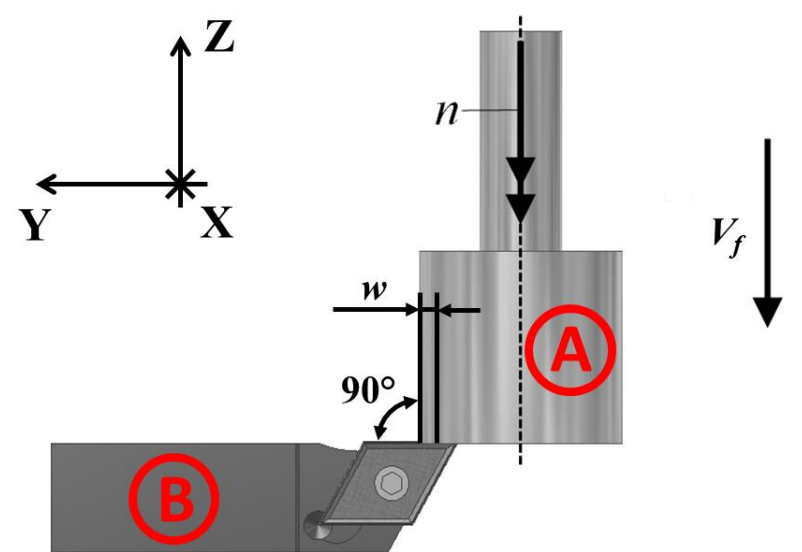

Fig. 4: Orthogonal cutting operation sketch [A = workpiece, $B=$ tool].

Each workpiece has been pre-machined on a traditional lathe and then measured on the Kern EVO by means of the Blum Micro Compact NT laser presetting system (accuracy: $1 \mu \mathrm{m}$ ) in order to detect possible errors. Diameter measurements on different workpiece - tool holder couples at different heights showed a maximum standard deviation $\sigma=7 \mu \mathrm{m}$ including dimensional, fixturing and run-out errors. Such a deviation is acceptable for the presented experimentation.

The orthogonal cutting tests were carried out by means of a Sandvik DCMX 070202 - WF1125 carbide insert (Fig. 5); the insert main characteristics are listed in Tab. 2.

The tested target material has been the 6082-T6 Aluminum alloy, whose measured hardness is 59 HRB. This material has been selected since it is the market currently available aluminum alloy similar to the Waldorf target material [9]. 
Tab.2: Experimental design constant parameters

\begin{tabular}{|l|l||l|}
\hline \multirow{4}{*}{ Insert } & Code & DCMX 070202 - WF1125 \\
\cline { 2 - 3 } & Nominal rake angle $(\alpha)$ & $18^{\circ}\left(\right.$ measured value $\left.\cong 16^{\circ}\right)$ \\
\cline { 2 - 3 } & Clearance angle & $7^{\circ}$ \\
\cline { 2 - 3 } & Cutting edge radius $\left(r_{\mathrm{e}}\right)$ & measured value $\cong 35 \mu \mathrm{m}$ \\
\hline \hline Target material & 6082-T6 Aluminum \\
\hline
\end{tabular}

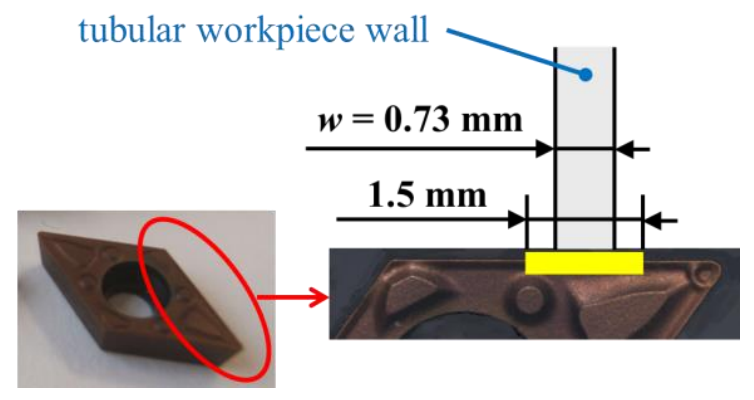

Fig. 5: Sandvik DCMX 070202 - WF1125 carbide insert.

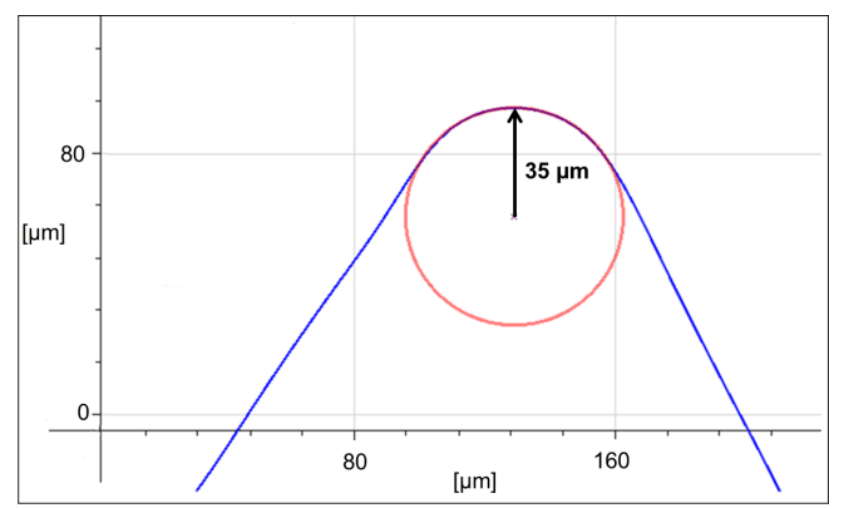

Fig. 6: Tool cutting edge radius measurement carried out by Alicona Infinite Focus (mean profile along yellow line in Fig. 5). 
The cutting edge radius and the rake angle have been measured by means of an Alicona Infinite Focus optical 3D measuring system (measurement parameters: 10x magnification, exposure time $=40.1 \mathrm{~ms}$, polarized light). The measured values are reported in Tab. 2. The cutting edge radius value (Fig. 6) has been obtained by calculating the mean of measurements made in three different zones along the insert cutting edge over a $1.5 \mathrm{~mm}$ length (yellow line in Fig. 5). The rake angle value cannot be shown on the profile of Fig. 6 where no references are reported.

Moreover, Alicona Infinite Focus has been used to measure the chip thickness $t$ directly on chips produced during each turning test. For each test, three chips have been measured three times over a very narrow band (white line in Fig. 7) to have a nearly punctual measurement (measurement parameters: 5x magnification, exposure time $=300 \mathrm{~ms}$, non-polarized light and ring light); the chip thickness value $t$ is the nine measurements mean. Chip thickness has also been checked by means of a Microrep DMS 680 Universal Length Measuring System.

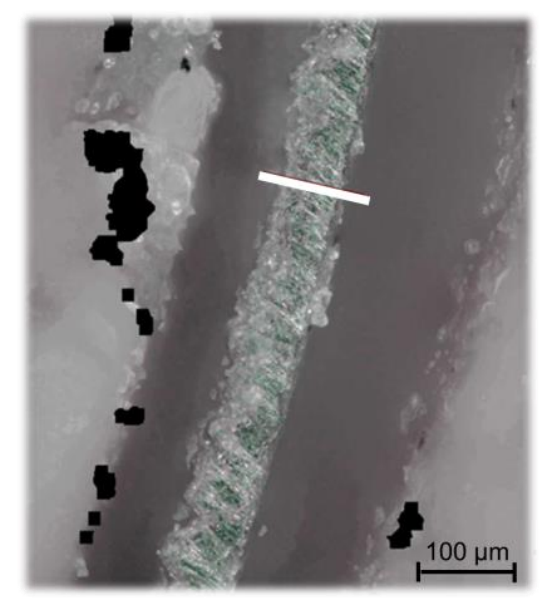

Fig. 7: Chip thickness measurement by Alicona Infinite Focus. 


\section{MODEL CALIBRATION AND FORCE PREDICTION PROCEDURE DEVELOPMENT}

The present study aims at demonstrating how the Waldorf's model is suitable for micromachining conditions. In order to achieve this result, this paper presents a clear and repeatable procedure for calibrating and applying a slip-line model to force prediction in the microscale. Analyzed literature lacks such a kind of procedure, making the model application very unstructured and leading to repeatability problems.

The scheme in Fig. 8 summarizes this procedure: boxes contain the reference to the applied formulas or actions to perform, while the arrows have different meanings depending on their position (box top and left: resources and input, box bottom and right: output).

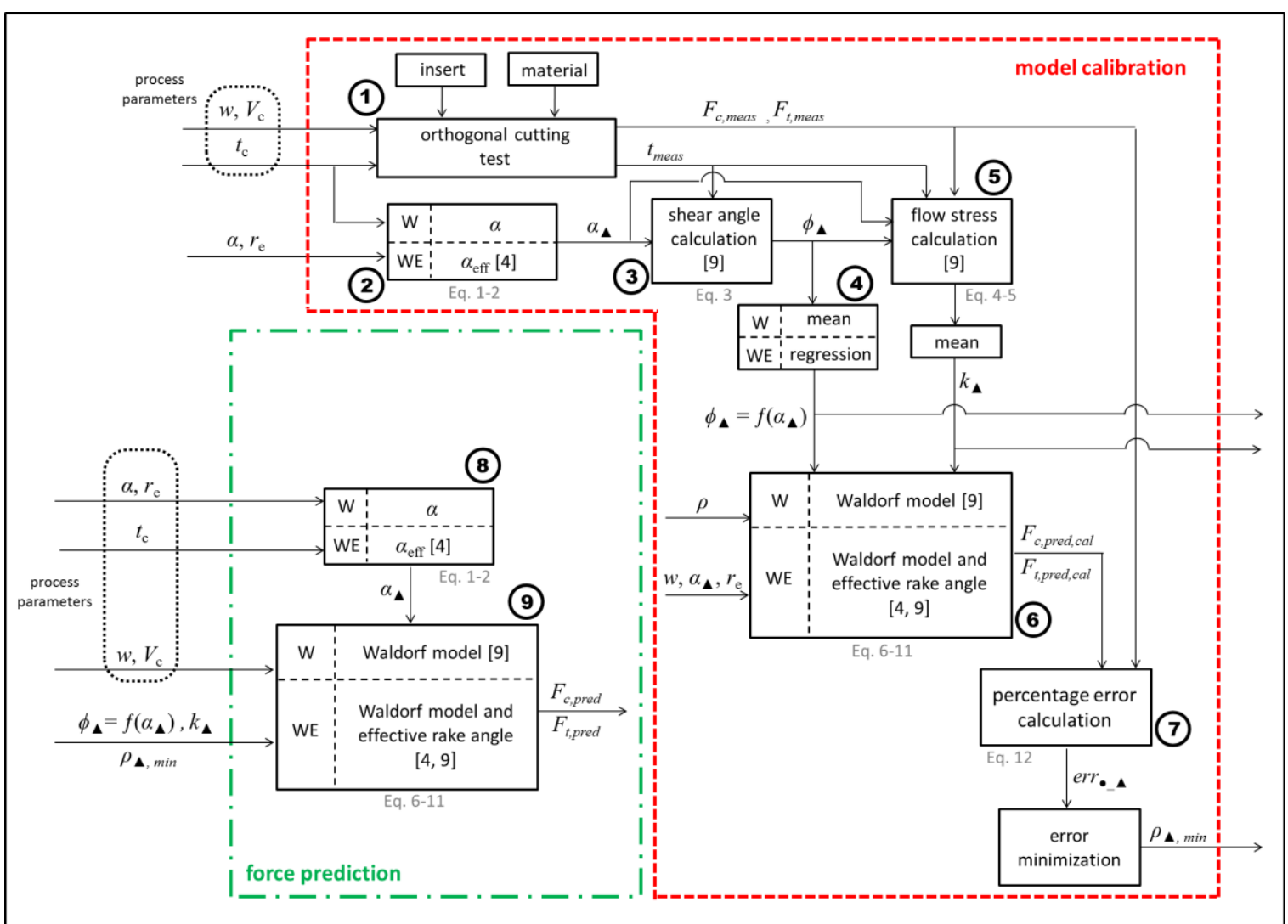

Fig. 8: Calibration and force prediction procedure scheme. 
The scheme in Fig. 8 is divided in two main parts, one concerning the model calibration phase and one concerning the model cutting force prediction phase. The whole procedure is applied to both the original Waldorf model and the Waldorf modified version considering the effective rake angle; since some quantities are different in the two cases, they have been indicated by the subscript $\boldsymbol{\Lambda}$, assuming the value "W" for the Waldorf model or "WE" for the Waldorf model modified with the effective rake angle. The comparison between the two model versions is a secondary result of the present study.

\subsection{Model Calibration}

The actions in the part of Fig. 8 named "model calibration" must be carried out prior to the model use, in order to calibrate it. The following part of this Section describes the scheme of Fig. 8 starting from box 1.

\section{Box 1 - Orthogonal cutting test}

Once the target material and the insert have been selected, some orthogonal cutting tests have to be performed with suitable process parameters. During the cutting tests, the cutting and thrust force components (respectively, $F_{\mathrm{c}}$ and $F_{\mathrm{t}}$ ) have to be acquired to calculate their mean value $\left(F_{\mathrm{c} \text {,meas }}, F_{\mathrm{t} \text {,meas }}\right)$. Moreover, the resulting chip thickness $t$ has to be measured for each experiment $\left(t_{\text {meas }}\right)$. It has to be pointed out as $F_{\mathrm{c} \text {,meas }}, F_{\mathrm{t} \text {,meas }}$ and $t_{\text {meas }}$ are vectors with lengths equal to the number of carried out calibration tests.

\section{Box 2 - Rake angle}

The rake angle $\alpha$ is equal to its nominal value when applying the $\mathrm{W}$ approach while it varies with the selected uncut chip thickness $t_{\mathrm{c}}$ when applying the effective rake angle approach. The effective rake angle $\alpha_{\text {eff }}$ (Fig. 9) is introduced in the Waldorf slip-line field model to account for typical low chip thickness effects on microcutting, which make the ploughing forces increase as the uncut chip thickness decreases. In particular, the effective rake angle introduction allows to model a dead metal cap size changing with the uncut chip thickness. 


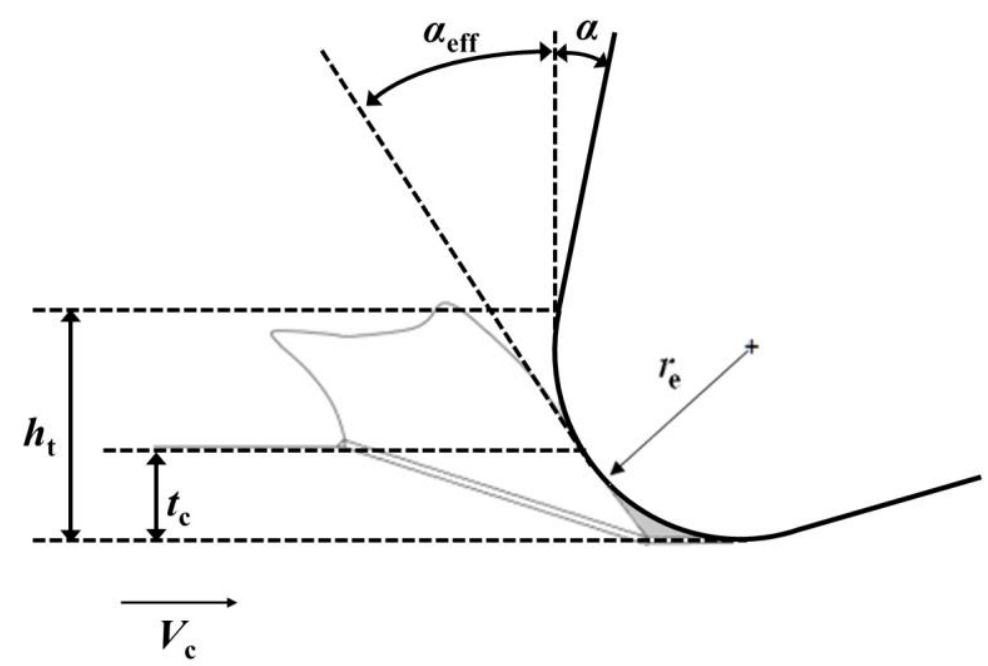

Fig. 9: Effective rake angle.

Eq. 1 [4] defines the height $h_{\mathrm{t}}$ of the tangency point between the tool rake surface and the cutting edge radius $r_{\mathrm{e}}$ arc.

$$
h_{\mathrm{t}}=r_{\mathrm{e}}(1+\sin (\alpha))
$$

When the uncut chip thickness $t_{\mathrm{c}}$ is lower than $h_{\mathrm{t}}$, the effective rake angle can be obtained as the inclination angle of the tangent to the cutter edge arc at the point corresponding to $t_{\mathrm{c}}$ (Eq. 2):

$$
\alpha_{\text {eff }}=-\frac{\pi}{2}+\cos ^{-1}\left(1-\frac{t_{\mathrm{c}}}{r_{\mathrm{e}}}\right)
$$

where $t_{\mathrm{c}} / r_{\mathrm{e}}$ is named "normalized uncut chip thickness". Otherwise, when the uncut chip thickness $t_{\mathrm{c}}$ is higher than $h_{\mathrm{t}}$, the effective rake angle $\alpha_{\mathrm{eff}}$ is equal to the nominal rake angle $\alpha$, as shown in Fig. 10, where three different nominal rake angle values are considered as an example. 


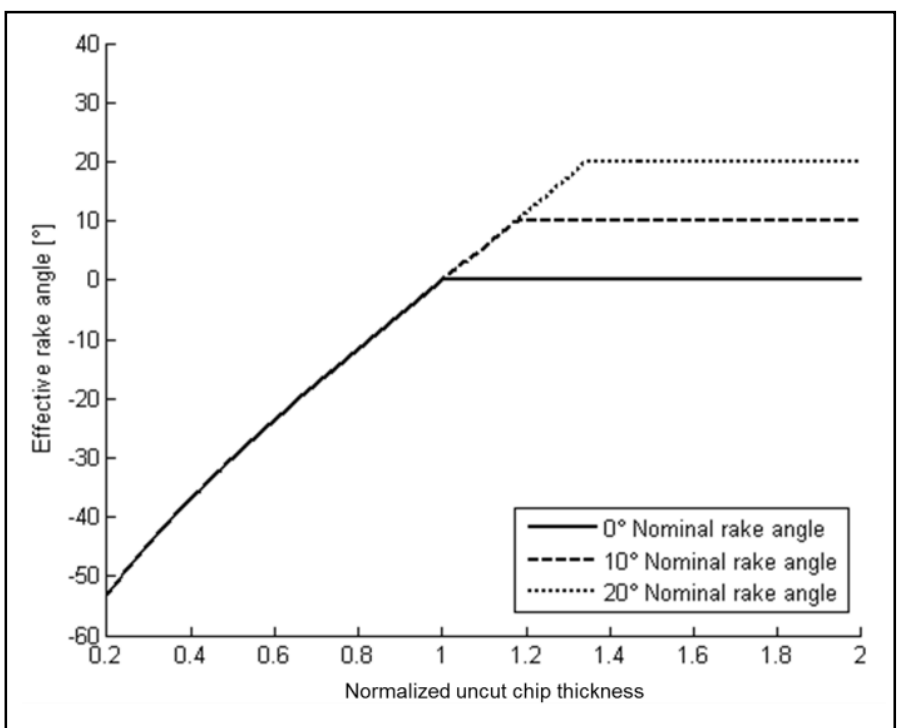

Fig. 10: Relationship between the effective rake angle $\alpha_{\text {eff }}$ and the normalized uncut chip thickness $t_{\mathrm{c}} / r_{\mathrm{e}}$

The box 2 output $\left(\alpha_{\mathbf{\Lambda}}\right)$ is a vector with length equal to the number of carried out calibration tests but, when applying the $\mathrm{W}$ approach, it is composed by identical elements equal to the nominal rake angle value.

\section{Box 3 - Shear angle (first step)}

A relation based on the chip compression ratio [9] is applied to calculate the shear angle $\phi$ :

$$
\tan \left(\phi_{\mathbf{\Lambda}}\right)=\frac{\frac{t_{\mathrm{c}}}{t_{\text {meas }}} \cos \left(\alpha_{\mathbf{\Delta}}\right)}{1-\frac{t_{\mathrm{c}}}{t_{\text {meas }}} \sin \left(\alpha_{\mathbf{\Delta}}\right)}
$$

Box 3 carries out a vector $\left(\phi_{\mathbf{\Lambda}}\right)$ with length equal to the number of performed calibration tests. 


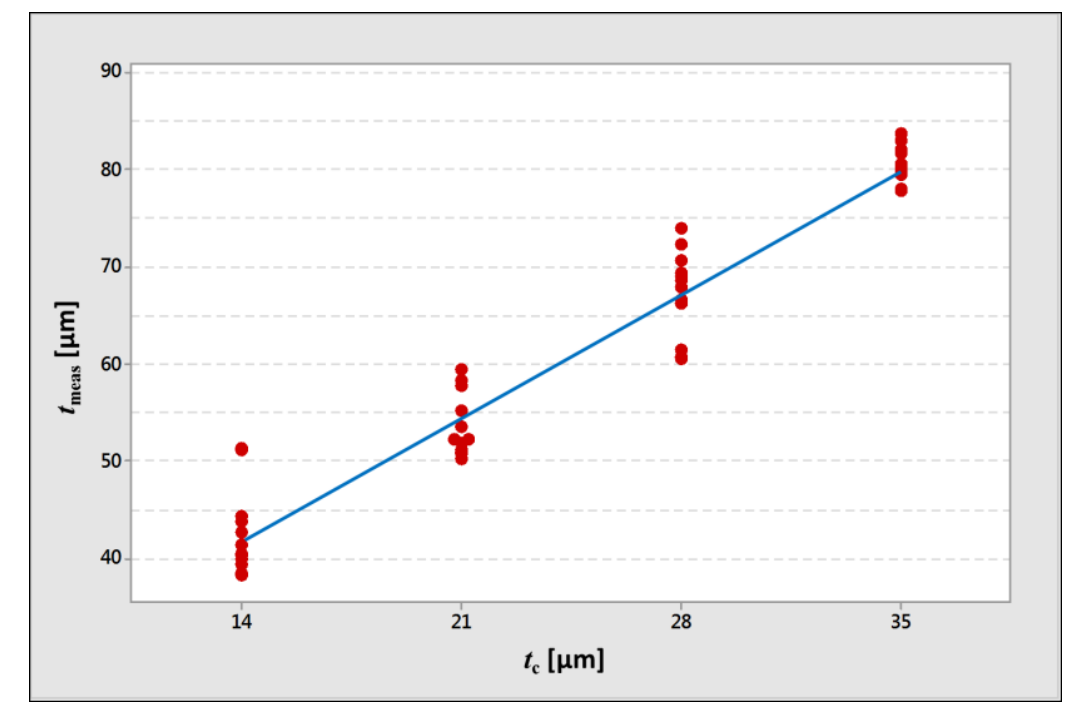

Fig. 11:Relationship between measured chip thickness $t_{\text {meas }}$ and uncut chip thickness $t_{\mathrm{c}}$

\section{Box 4 - Shear angle (second step)}

The $t_{c} / t_{\text {meas }}$ ratio can be assumed to be almost constant as observed from experimental data (Fig. 11), therefore the shear angle $\phi$ only depends on the rake angle $\alpha$. If $\alpha$ is constant, as according to the $\mathrm{W}$ approach, $\phi$ is also constant so it is reasonable to calculate the mean value of $\phi_{\mathrm{W}}$ obtained data (box $4, \mathrm{~W}$ approach, in Fig. 8). In this first case, the box 4 output is a scalar. On the other hand, when considering the effective rake angle (WE approach), $\phi_{\mathrm{WE}}$ is proportional to $\alpha_{\mathrm{eff}}$ and their relationship can be found by means of a regression analysis, as done in [24, 33] (box 4, WE approach, in Fig. 8). Fig. 12 shows how a linear relationship can fit data, as a proof of the correct assumption of constant $t_{c} / t_{\text {meas }}$ ratio. In this second case, the box 4 output is a function.

Fig. 12 represents all the 48 experimental results making part of the experimental database carried out in this study (Tab. 1) in order to demonstrate the correctness of the described assumptions on the shear angle 
dependence from the rake angle and the chip thickness. The calibration procedure does not need such an amount of data to be effective, as it has been pointed out in Section 5 .

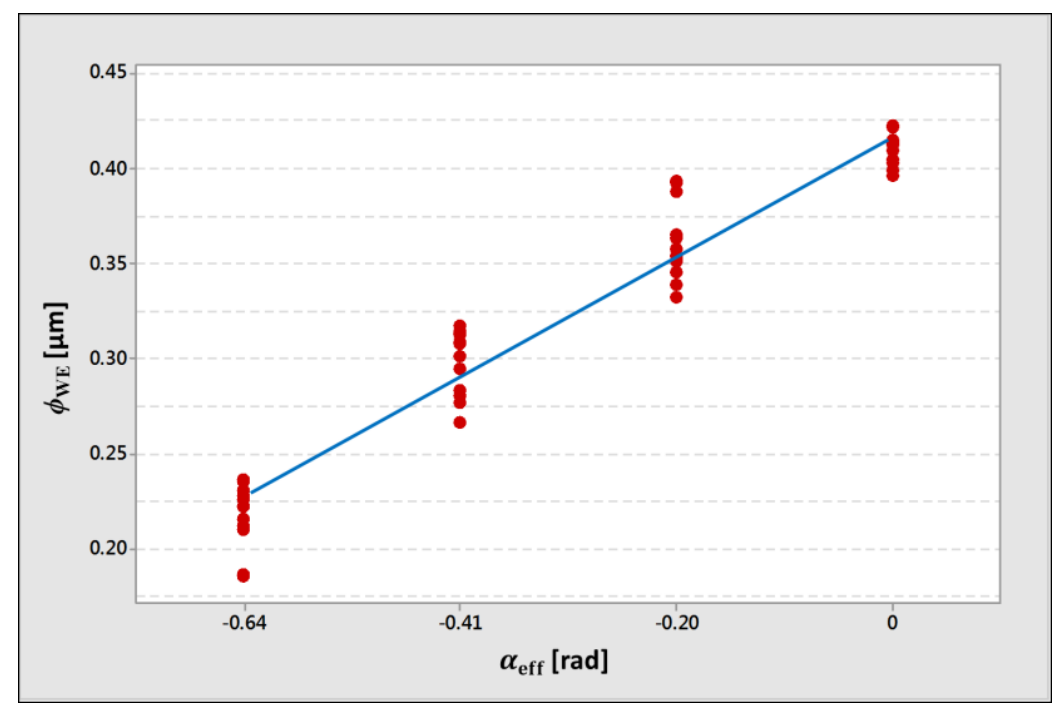

Fig. 12: Relationship between shear angle $\phi_{\mathrm{WE}}$ and effective rake angle $\alpha_{\mathrm{eff}}(\mathrm{WE}$ approach).

\section{Box 5 - Flow stress}

The flow stress $k$ for the considered target material is calculated for each cutting test by applying the following equation [9]:

$$
k=\frac{F_{\mathrm{c}, \mathrm{meas}} \cos \left(\phi_{\mathbf{\Lambda}}\right)-F_{\mathrm{t} \text {,meas }} \sin \left(\phi_{\mathbf{\Lambda}}\right)}{w \cdot \mathrm{AB}}
$$

where the shear plane length is evaluated as follows [9]:

$$
\mathrm{AB}=\frac{t_{\text {meas }}}{\cos \left(\phi_{\mathbf{\Delta}}-\alpha_{\mathbf{\Delta}}\right)}
$$


Since $k$ is supposed to be independent from cutting parameters, the value to use as force prediction model input is obtained as the mean of calculated values for both the W and the WE approaches. For this reason, the box 5 gives a scalar as output.

Eq. 4 [9] only works when the ploughing forces are negligible, i.e. for higher values of normalized uncut chip thickness, which does not represent a typical microscale condition. Since the proposed procedure is modular, each box can be changed independently from the others. In particular, the flow stress $k$ could be calculated according to other methods, such as the Johnson-Cook model or the relationship between the flow stress and the microhardness [37]. This opportunity will be evaluated in future developments of the present research.

\section{Box 6 - Prow angle}

Before using the model to predict cutting forces, also the prow angle $\rho$ (Fig. 1) has to be calibrated. In order to do that, $F_{\mathrm{c}}$ and $F_{\mathrm{t}}$ can be calculated for different $\rho$ values basing on the already obtained $\phi_{\boldsymbol{\Lambda}}$ and $k_{\boldsymbol{\Lambda}}$ parameters.

Box 6 applies the following equations (6-10) [9], where $\rho$ is a vector of possible prow angle values (in this paper, 15 values ranging from 0 to $0.7 \mathrm{rad}$ with steps of $0.05 \mathrm{rad}$ have been tested) and $F_{\mathrm{c} \text {,pred,cal }}, F_{\mathrm{t} \text {,pred,cal }}$ are matrixes whose dimensions correspond to the number of carried out calibration tests and to the $\rho$ vector length.

$$
\begin{aligned}
& F_{\text {c.,pred,cal }}=k_{\mathbf{\Lambda}} \cdot w \cdot\left\{\begin{array}{l}
{\left[\cos \left(\phi_{\mathbf{\Delta}}\right)+(1+2 \theta) \cdot \sin \left(\phi_{\mathbf{\Delta}}\right)\right] \cdot \mathrm{AB}} \\
+\left[\cos (2 \eta) \cdot \cos \left(\phi_{\mathbf{\Delta}}-\gamma+\eta\right)+(1+2 \theta+2 \gamma+\sin (2 \eta)) \cdot \sin \left(\phi_{\mathbf{\Lambda}}-\gamma+\eta\right)\right] \cdot \mathrm{CA}
\end{array}\right\} \\
& F_{\text {t.pred,cal }}=k_{\mathbf{\Lambda}} \cdot w \cdot\left\{\begin{array}{l}
{\left[(1+2 \theta) \cdot \cos \left(\phi_{\mathbf{\Delta}}\right)-\sin \left(\phi_{\mathbf{\Delta}}\right)\right] \cdot \mathrm{AB}} \\
+\left[(1+2 \theta+2 \gamma+\sin (2 \eta)) \cdot \cos \left(\phi_{\mathbf{\Delta}}-\gamma+\eta\right)-\cos (2 \eta) \cdot \sin \left(\phi_{\mathbf{\Lambda}}-\gamma+\eta\right)\right] \cdot \mathrm{CA}
\end{array}\right\}
\end{aligned}
$$

where:

$$
\theta=\frac{\pi}{4}-\rho-\phi_{\Delta}
$$




$$
\begin{aligned}
& \eta=0.5 \cdot \cos ^{-1}(m) \\
& \gamma=\eta+\phi_{\mathbf{\Delta}}-\sin ^{-1}(\sqrt{2} \cdot \sin (\rho) \cdot \sin (\eta)) \\
& \mathrm{CA}=\frac{R}{\sin (\eta)}
\end{aligned}
$$

The radius $R$ of the circular fan field centred in A (Fig. 1) is obtained by solving Eq. 11 [9].

$$
R=\sin (\eta) \sqrt{\left(r_{\mathrm{e}} \cdot \tan \left(\frac{\pi}{4}+\frac{\alpha_{\mathbf{\Delta}}}{2}\right)+\frac{\sqrt{2} \cdot R \cdot \sin (\rho)}{\tan \left(\frac{\pi}{4}+\alpha_{\mathbf{\Delta}}\right)}\right)^{2}+2[R \cdot \sin (\rho)]^{2}}
$$

\section{Box 7 - Percentage error}

The percentage errors between the force predicted values $\left(F_{\mathrm{c}, \text { pred,cal }}\right.$ and $\left.F_{\mathrm{t}, \text { pred,cal }}\right)$ and the measured mean values $\left(F_{\mathrm{c}, \text { meas }}\right.$ and $\left.F_{\mathrm{t}, \text { meas }}\right)$ can be calculated as follows:

$$
\operatorname{err} \boldsymbol{\bullet} \_\mathbf{\Delta}=\left|\frac{F_{\cdot \text {,pred } \mathbf{\Delta}}-F_{\cdot \text {,meas }}}{F_{\bullet \text {,meas }}}\right| \cdot 100
$$

where $\bullet$ describes the force direction assuming the value "c" for cutting or "t" for thrust force.

Each $e r r_{\bullet} \_$is a matrix with the same dimensions as $F_{\text {c,pred,cal }}$ and $F_{\text {t,pred,cal }}$ matrixes.

For each err._ matrix, the mean of the percentage errors obtained for each prow angle value is calculated; the result is a vector of mean errors whose length corresponds to the number of possible prow angle values. The optimal value of the prow angle $\rho_{\mathbf{\Delta}, \min }$ (which is a scalar) is the one minimizing the maximum of the cutting and thrust force mean percentage errors.

Once the models have been completely calibrated, they can be used to predict cutting forces for the required process parameters (“force prediction" part of Fig. 8). 


\subsection{Force Prediction}

This section briefly explains the "force prediction" part of the Fig. 8 scheme. As it can be noticed, it is composed of two boxes (box 8 and box 9) that correspond, respectively, to box 2 and box 6 of the "model calibration" part.

In particular, the box 8 is completely identical to box 2 and calculates the rake angle according to both the $\mathrm{W}$ and the WE approaches. Box 9 is composed by the same equations of box 6 ; regarding its input, it receives $\phi_{\mathbf{\Delta}}=f\left(\alpha_{\mathbf{\Delta}}\right), k_{\mathbf{\Delta}}$ and $\rho_{\mathbf{\Delta} \text {,min }}$ values from the calibration procedure and $\alpha_{\mathbf{\Delta}}$ from the box 8 , where the model user has to provide $\alpha, r_{\mathrm{e}}$, and $t_{\mathrm{c}}$ as input. The user has also to provide $w$ and $V_{\mathrm{c}}$ as a direct input of box 9 .

Once the tool is defined ( $\alpha$ and $r_{\mathrm{e}}$ parameters), the model predicts the cutting and thrust mean values $\left(F_{\mathrm{c}, \text { pred }}\right.$ and $\left.F_{\mathrm{t}, \mathrm{pred}}\right)$ for each combinations of process parameters $t_{\mathrm{c}}, V_{\mathrm{c}}$ and $w$. In this case, $F_{\mathrm{c}, \text { pred }}$ and $F_{\mathrm{t}, \text { pred }}$ are scalars since the "force prediction" part works on a single experimental condition at a time.

\section{MODEL CALIBRATION CONDITIONS SELECTION}

The calibration procedure has been performed using three different and representative experimental sets (shown in Fig. 13 by the square ( $\mathbf{\square})$, star $(\star)$ and diamond ( $)$ symbols) among the available combinations coming from the carried out experimental design (Tab. 1). In the case of this paper, each set consists of 8 runs, since 4 replicates have been performed for both of the 2 considered experimental conditions. The considered experimental calibration conditions must have a different normalized uncut chip thickness in order to allow the calibration procedure to estimate the linear relationship between the shear angle $\phi_{\mathbf{A}}$ and the rake angle $\alpha_{\mathbf{\Delta}}\left[\phi_{\mathbf{\Lambda}}=f\left(\alpha_{\mathbf{\Lambda}}\right)\right]$. Since the proposed procedure aims at reducing the experimental effort needed for the model calibration, the minimum number of experimental conditions (i.e. two) has been selected. 


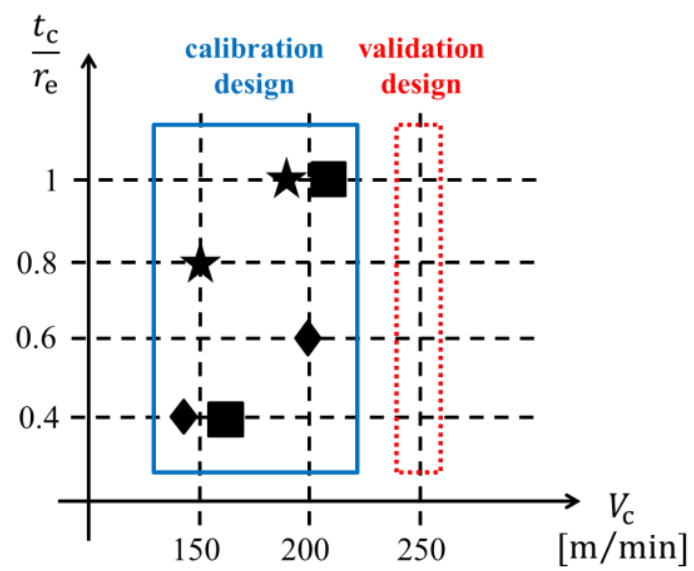

Fig. 13: Experimental sets for the model calibration.

Once the calibration has been carried out, both the original Waldorf model (W) and the Waldorf model considering the effective rake angle (WE) have been applied to predict forces for all the 8 combinations of $t_{\mathrm{c}} / r_{\mathrm{e}}$ and $V_{\mathrm{c}}$ of the calibration design (Tab. 1), so also for combinations outside the calibration set. This procedure can be considered a first validation of the model for different normalized uncut chip thickness values respect to the calibration ones. Eventually, percentage errors have been calculated according to Eq. 12. 


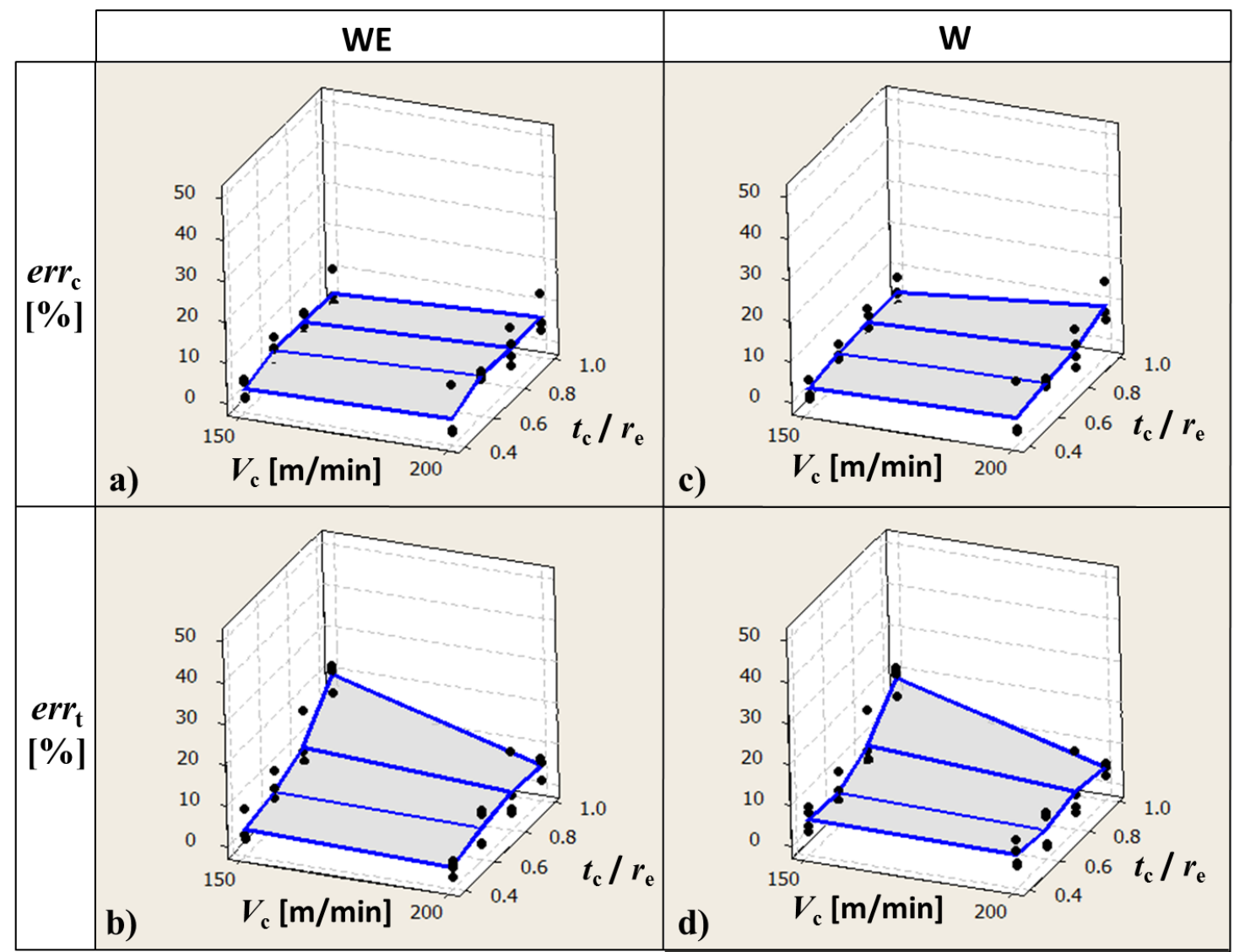

Fig. 14: Percentage errors for the star ( $\star$ calibration set [a) $\left.\left.\left.\left.\operatorname{err}_{\mathrm{c}_{-} \mathrm{WE}}, b\right) e r r_{\mathrm{t}_{-} \mathrm{WE}}, c\right) e r r_{\mathrm{c}_{-} \mathrm{W}}, d\right) e r r_{\mathrm{t}_{-} \mathrm{W}}\right]$. 


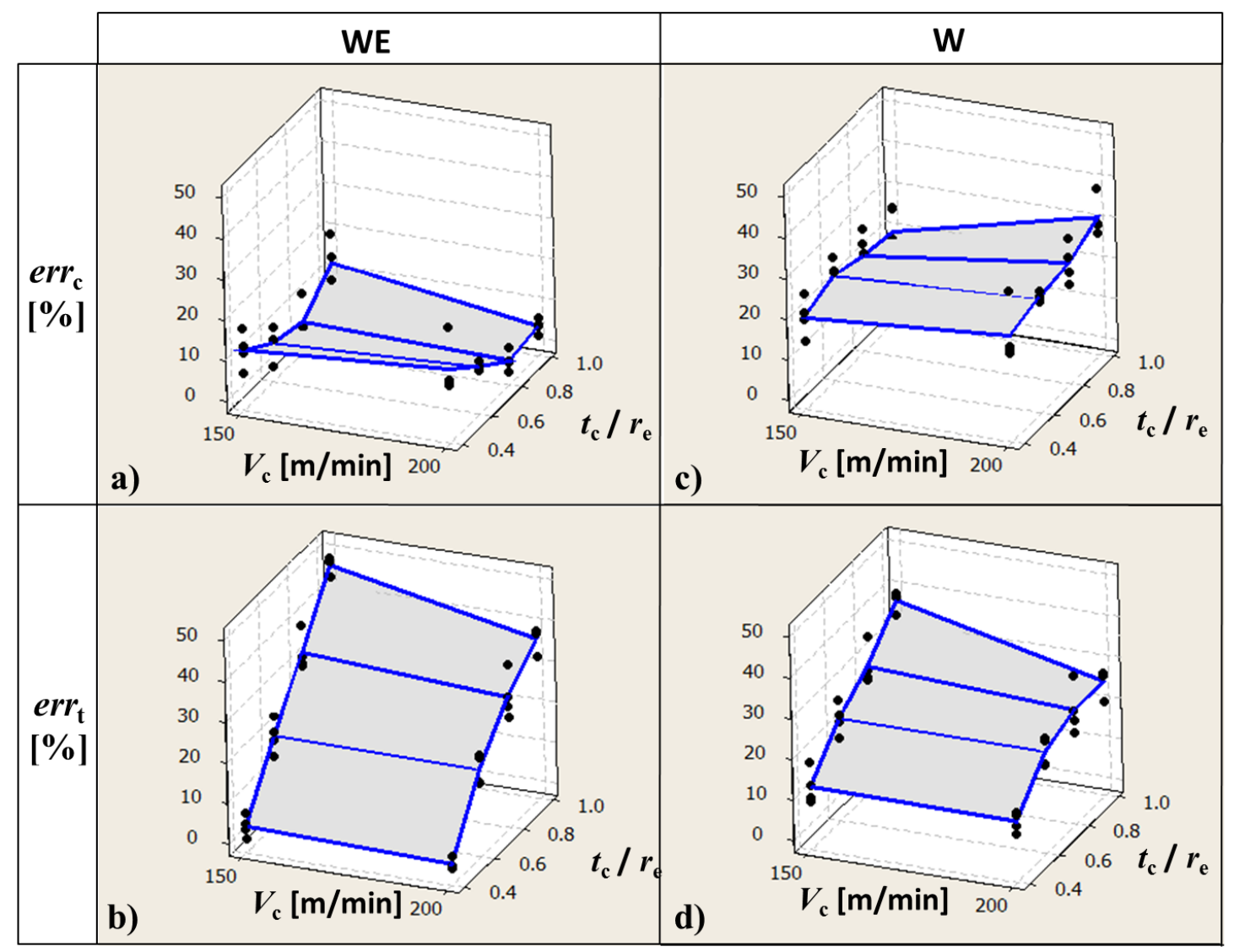

Fig. 15: Percentage errors for the diamond ( ) calibration set [a) $\left.\left.\left.e r r_{\mathrm{c}_{-} \mathrm{WE}}, b\right) e r r_{\mathrm{t}_{-} \mathrm{WE}}, c\right) e r r_{\mathrm{c}_{-} \mathrm{W}}, d\right)$ $\left.e r r_{t_{-}}\right]$. 


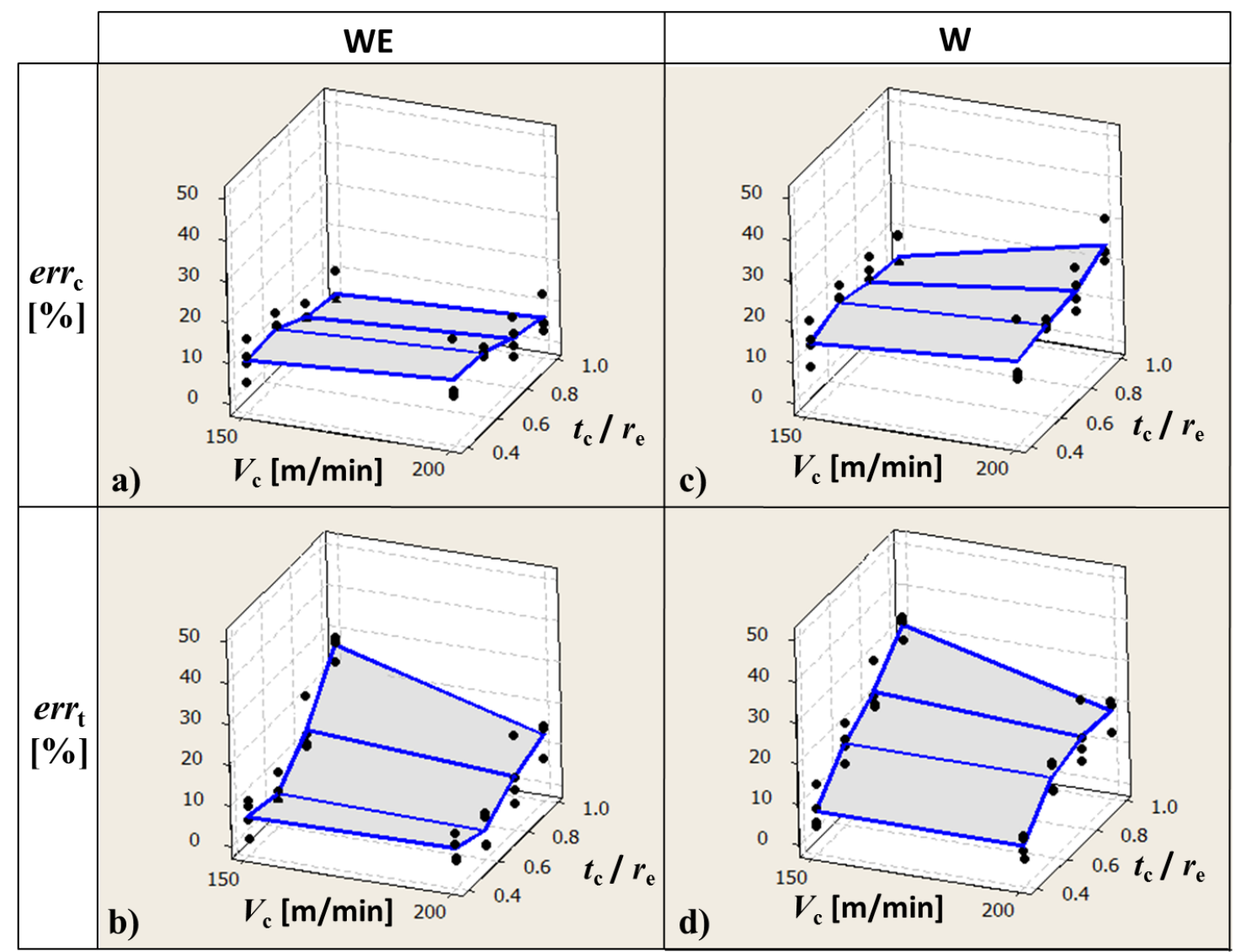

Fig. 16: Percentage errors for the square (অ) calibration set [a) $\left.\left.\left.\left.\operatorname{err}_{\mathrm{c}_{-} \mathrm{WE}}, b\right) e r r_{\mathrm{t}_{-} \mathrm{WE}}, c\right) e r r_{\mathrm{c}_{-} \mathrm{w}}, d\right) e r r_{\mathrm{t}_{-} \mathrm{w}}\right]$.

Both the models improve their prediction performance passing from diamond $(\checkmark)$ to square $(\boldsymbol{\square})$ and, finally, to star $(\star)$ calibration set. WE model is better in almost all the cases, except for the thrust force error with the diamond ( ) calibration set; in particular, it is possible to notice (Fig. 14) how the model performances in case of both $\mathrm{W}$ and WE approaches are comparable for the star $(\star)$ calibration set. This result suggests to calibrate both models for normalized uncut chip thicknesses close to unity, i.e. before the point where the slope of the effective rake angle curve (Fig. 10) changes and the effective rake angle $\alpha_{\text {eff }}$ becomes similar to the nominal rake angle $\alpha$. 
The strength of the proposed WE approach is that the model, once calibrated with the best condition $(\star)$, better predicts forces, in particular where the uncut chip thickness is low (percentage errors are less than $20 \%$ for $\left.t_{\mathrm{c}} / r_{\mathrm{e}}=0.4-0.6\right)$.

\section{MODEL VALIDATION}

The model prediction performance has been validated by means of the validation design experiments (red dotted box in Fig 17). All the experimental conditions in this set have a different cutting speed than those in the calibration design (blue solid box in Fig 17) in order to prove the model robustness to the cutting speed effects; in fact, the cutting speed is not a model input parameter. The cutting speed for the validation design experiments has been set to $250 \mathrm{~m} / \mathrm{min}$ to maintain a constant difference among the cutting speed levels. Moreover, when machining in the microscale, it is difficult to reach higher cutting speeds due to the limitations on maximum spindle speeds.

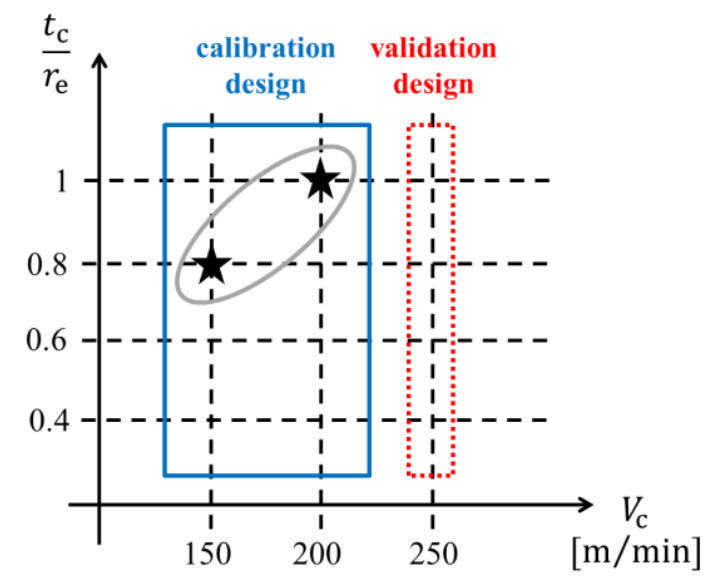

Fig. 17: Validation with the best calibration set $(\star)$. 
Both the W and the WE model, calibrated with the best calibration set $(\star)$, have been applied to predict the cutting forces for the validation design experimental conditions and the percentage errors have been calculated according to Eq. 12.

Fig. 18 shows the model prediction performance both in the calibration set $(\star)$ and in all the other tested experimental conditions (i.e. the remaining 10 combinations of $t_{\mathrm{c}} / r_{\mathrm{e}}$ and $V_{\mathrm{c}}$ that are not indicated by the stars in Fig. 17). Basically, the calibration design has been used to select the calibration conditions; once the model has been calibrated with the best set $(\star)$, all the other experimental conditions (both in the calibration design and in the validation design) are used to evaluate the model prediction performance, thus acting all as validation tests. 


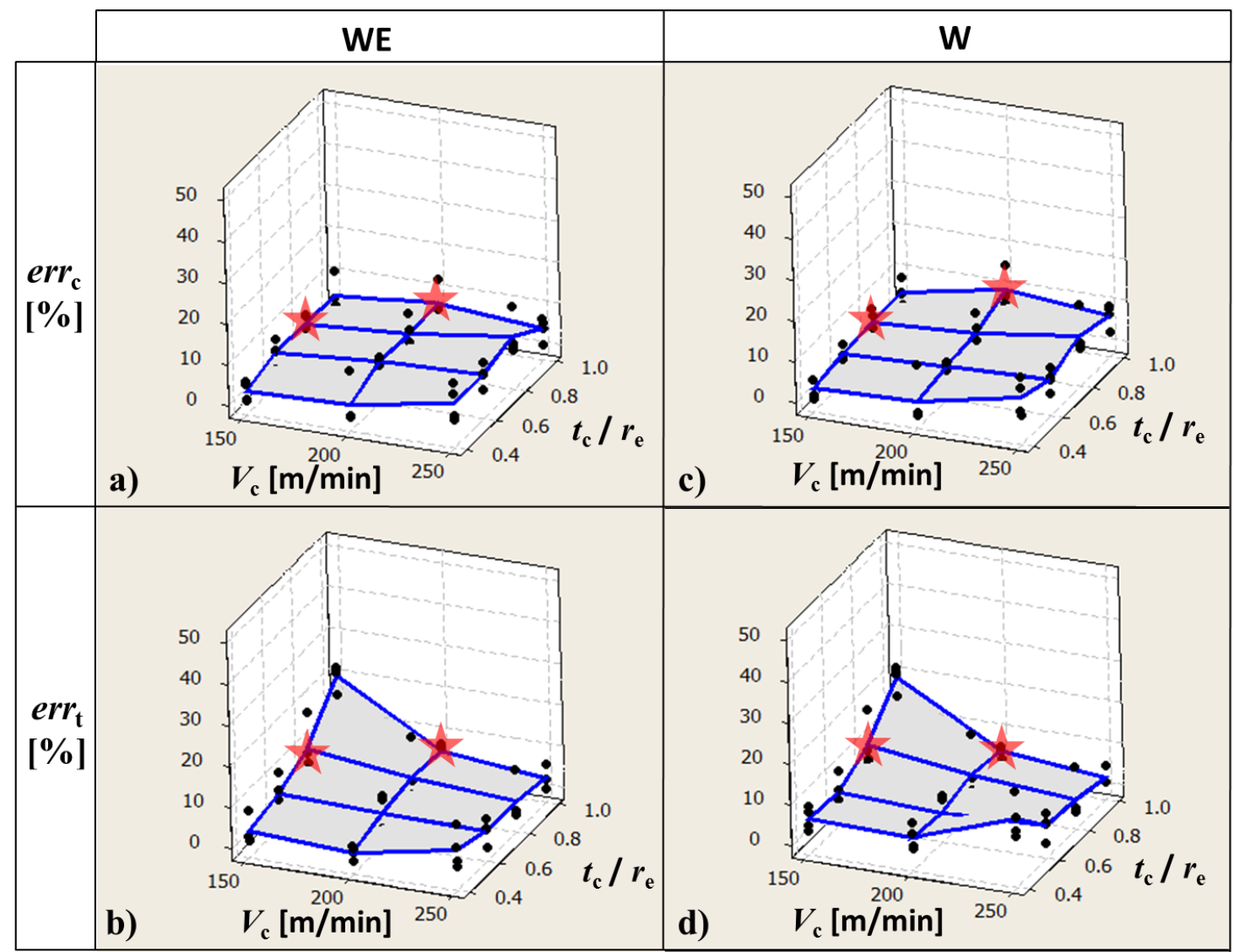

Fig. 18: Percentage errors for the star ( $\star$ ) calibration set comprising the validation experimental condition [a) $e r r_{\mathrm{c}_{-} \mathrm{WE}}$, b) $\left.\left.\left.e r r_{\mathrm{t}_{-} \mathrm{WE}}, c\right) e r r_{\mathrm{c}_{-} \mathrm{W}}, d\right) e r r_{\mathrm{t}_{-} \mathrm{W}}\right]$.

Fig. 18 shows how the WE model prediction performance is better than the $\mathrm{W}$ model prediction performance in the calibration design space and is good also for the experimental conditions of the validation design. This fact highlights how the WE model, once correctly calibrated, is able to accurately predict the cutting forces outside the calibration window. Moreover, the model validation with a cutting speed outside the calibration set proves its robustness to cutting speed effects. In fact, the cutting speed is 
not a model input parameter, but it is known that the cutting speed affects the material properties (e.g. a cutting speed increase causes a softening effect).

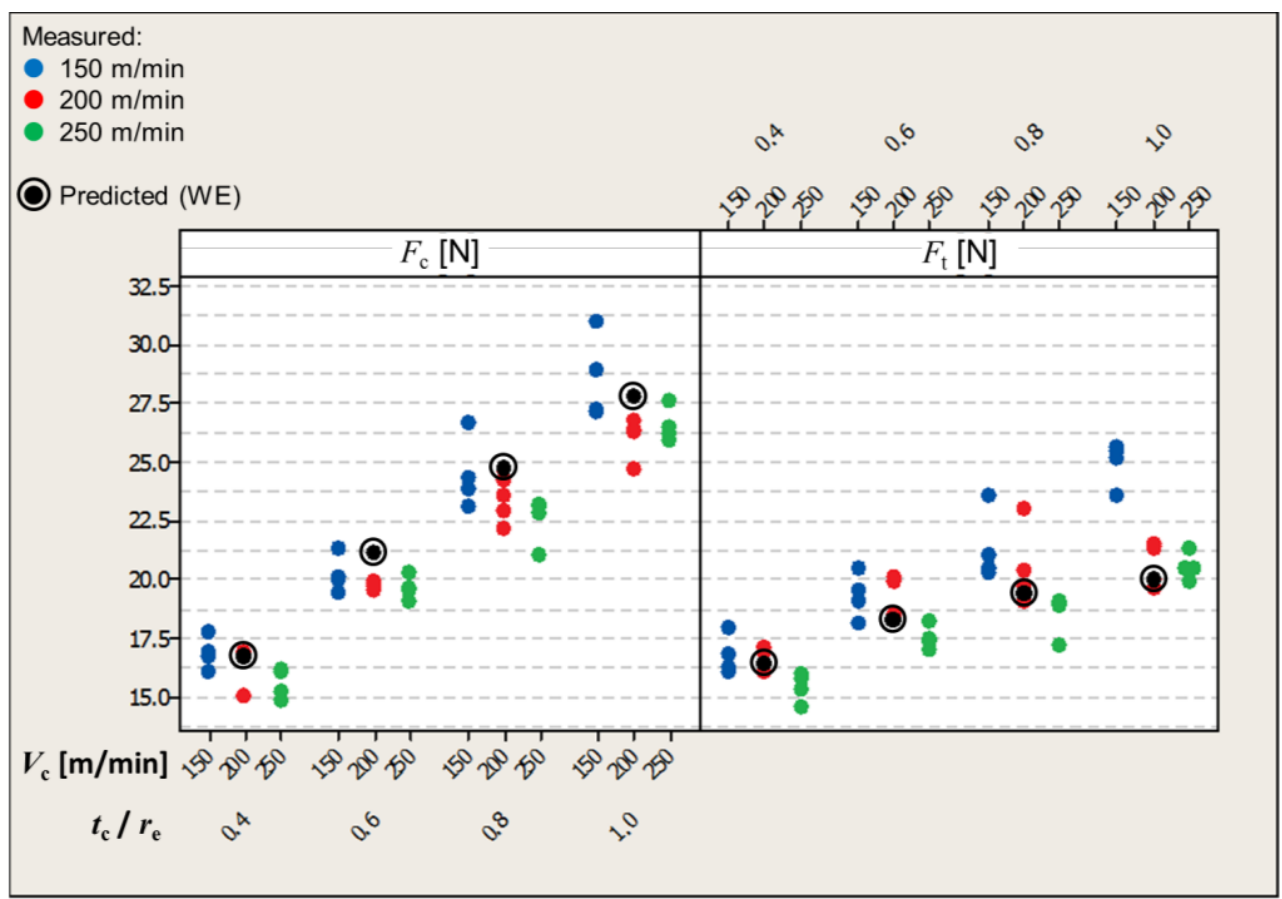

Fig. 19: Measured and predicted (WE approach) values of cutting and thrust forces for the star ( $\star$ ) calibration set.

For the sake of completeness, Fig. 19 shows the cutting and thrust force values which have been measured for all the cutting trials in the experimental database (Tab. 1) and the values which have been predicted applying the WE approach in case of the best calibration set ( $\star$ ). 


\section{CONCLUSIONS AND FUTURE DEVELOPMENTS}

The present paper has been focused on the Waldorf's slip-line field model [9-10] applied to typical microcutting parameters in both its original version and a modified version implementing the effective rake angle [4]. The purpose has been to demonstrate the model adequacy to predict forces in the microfield. In order to achieve this goal, a clear, modular, objective and repeatable procedure making the selected slip-line field model an applicable and sustainable force prediction instrument in the microscale has been developed.

The model modified version is generally better for the tested calibration conditions but, in particular, at low uncut chip thickness where the model seems to work satisfactorily in the microfield. Such an objective conclusion and the carried out calibration and prediction procedures are important for future developments.

Next studies will aim at substituting some procedure modules (Fig. 8) to extend the calculation validity overcoming some restrictive assumptions, e.g. in the case of the flow stress $k$ calculation (box 5).

Further studies will deal with other target materials and other tool geometries in order to objectively check the model possible extensions. A structured quantitative criterion will be obtained for the calibration condition selection. Moreover, further validation tests for other experimental conditions outside the original experimental design will be carried out in order to extend the present paper results.

The model will also be applied to predict shear and ploughing forces and a suitable validation, based on chip formation micrographs, will be carried out for this purpose.

\section{ACKNOWLEDGMENTS}

This study has been partly founded by Regione Lombardia within the project "REMS: Rete Lombarda di Eccellenza per la Meccanica Strumentale e Laboratorio Esteso / Excellence Network for Instrumental Mechanics and Extended Laboratory” (Fondo per la promozione di Accordi Istituzionali, D. Reg. n4779 del 14/5/2009). 


\section{REFERENCES}

1. Liu X, DeVor RE, Kapoor SG, et al. The Mechanics of Machining at the Microscale: Assessment of the Current State of the Science. J Manuf Sci Eng-Trans ASME 2004; 126 (11):666-678.

2. Dornfeld D. Recent Advances in Mechanical Micromachining. CIRP Ann-Manuf Technol 2006; 55(2): 745-768.

3. Weule H, Huntrup V and Tritschle H. Micro-Cutting of Steel to Meet New Requirements in Miniaturization. CIRP Ann-Manuf Technol 2001; 49(1): 61-64.

4. Lee HU, Cho DW and Ehmann KF. A Mechanistic Model of Cutting Forces in Micro-End-Milling with Cutting-Condition-Independent Cutting Force Coefficients. J Manuf Sci Eng-Trans ASME 2008; $130(3)$.

5. Kim CJ, Bono M and Ni J. Experimental Analysis of Chip Formation in Micro-Milling Trans. NAMRI/SME, 2002; 30: 247-254.

6. Kim CJ, Mayor JR and Ni J. A Static Model of Chip Formation in Microscale Milling J Manuf Sci Eng-Trans ASME 2004: 126(11): 710-718.

7. Vogler MP, DeVor RE and Kapoor SG. Microstructure-Level Force Prediction Model for Micro-Milling of Multi-Phase Materials J Manuf Sci Eng-Trans ASME 2003; 125(2): 202-209.

8. Lawson BL, Kota $\mathrm{N}$ and Ozdoganlar OB. Effects of Crystallographic Anisotropy on Orthogonal Micromachining of Single-Crystal Aluminum J Manuf Sci Eng-Trans ASME 2008; 130(3).

9. Waldorf DJ, DeVor RE and Kapoor SG. A Slip-Line Field for Ploughing during Orthogonal Cutting $J$ Manuf Sci Eng-Trans ASME 1998; 120(4): 693-698.

10. Waldorf DJ, DeVor RE and Kapoor SG. An Evaluation of Ploughing Models for Orthogonal Machining J Manuf Sci Eng-Trans ASME 1999; 121(4): 550-558.

11. Kountanya RK and Endres WJ. A High-Magnification Experimental Study of Orthogonal Cutting with Edge-Honed Tools In: ASME International Mechanical Engineering Congress and Exposition - IMECE 
New York, USA, 11-16 November 2001, Paper No. IMECE2001/MED-23317.

12. Lee EH and Shaffer BW. The Theory of Plasticity applied to a Problem of Machining Trans ASME 1951; 18: 405-413.

13. Oxley PLB. The Mechanics of Machining: an Analytical Approach to Assessing Machinability Chichester: Ellis Horwood Limited, 1989.

14. Kudo H. Some New Slip-Line Solutions for Two Dimensional Steady-State Machining Int J Mech Sci $1965 ; 7: 43-55$.

15. Dewhurst P. On the Non-Uniqueness of the Machining Process Proc Royal Society of London 1997; 360: $587-610$.

16. Maity KP and Das NS. A Class of Slip-Line Field Solutions for Metal Machining with Elastic Contact J Mater Process Technol 1998; 96(1): 9-18.

17. Toropov A and Ko SL. A New Slip-Line Theory for Orthogonal Cutting and its Application. In: $3^{\text {rd }}$ International Asia Pacific Forum on Precision surface finishing, 2001.

18. Liu XD, Lee LC and Lam KY. A Slip-Line Field Model for the Determination of Chip Curl Radius $J$ Eng Ind-Trans ASME 1995; 117: 266-271.

19. Fang N, Jawahir IS and Oxley PLB. A Universal Slip-Line Model with Non-Unique Solutions for Machining with Curled Chip Formation and a Restricted Contact Tool Int J Mech Sci 2001, 43(2): 557-580. 20. Dundur ST and Das NS. Slipline Field Modeling of Orthogonal Machining for a Worn Tool with Elastic Effects and Adhesion Friction at the Contact Region J Mater Process Tech 2009; 209(1): 18-25.

21. Fang N. Slip-Line Modeling of Machining with a Rounded-Edge Tool - Part I: New Model and Theory J Mech Phys Solids 2003; 51(4): 715-742.

22. Fang N. Slip-Line Modeling of Machining with a Rounded-Edge Tool - Part II: Analysis of the Size Effect and The Shear Strain-Rate J Mech Phys Solids 2003; 51 (4): 743-762. 
23. Jun MBG, Liu M, DeVor RE. et al. Investigation of the Dynamics of Microend Milling-Part I: Model Development J Manuf Sci Eng-Trans ASME 2006; 128(4): 893-900.

24. Liu M, Jun MBG, DeVor RE, et al. Cutting Mechanisms and their Influence on Dynamic Forces, Vibrations and Stability in Micro-Endmilling. In: ASME International Mechanical Engineering Congress and Exposition - IMECE Anaheim, California, USA, 13 - 19 November 2004, paper $\mathrm{n}^{\circ}$ IMECE2004-62416, pp. 583-592

25. Liu M, DeVor RE and Kapoor SG. An Analytical Model for the Prediction of Minimum Chip Thickness in Micromachining J Manuf Sci Eng-Trans ASME 2006; 128(2): 474-481.

26. Karpat Y and Özel T Mechanics of High Speed Cutting with Curvilinear Edge Tools Int J Mach Tools Manuf 2008; 48(2): 195-208.

27. Yoon HS and Ehmann KF. A Slip-Line Field Model for Orthogonal Micromachining Processes. In: 4M/ICOMM Conference, Karlsruhe, Germany, 23-25 September 2009, pp. 329-332.

28. Jin X and Altintas Y. Slip-Line Field Model of Micro-Cutting Process with Round Tool Edge Effect $J$ Mater Process Tech 2011; 211(3): 339-355.

29. Ozturk S and Altan E. A Slip-Line Approach to the Machining with Rounded-Edge Tool Int J Adv Manuf Technol 2012; 63(5-8): 513-522.

30. Ren H and Altintas Y. Mechanics of Machining with Chamfered Tools J Manuf Sci Eng-Trans ASME 2000; 122(4): 650-659.

31. Long Y and Huang Y. Force Model under Dead Metal Zone Effect in Orthogonal Cutting with Chamfered Tools Trans. NAMRI/SME 2005; 33: 573-580.

32. Shi T and Ramalingam S. Slip-Line Solution for Orthogonal Cutting with a Chip Breaker and Flank Wear Int J Mech Sci 1991; 33(9): 689-704.

33. Vogler MP, DeVor RE and Kapoor SG. On the Modeling and Analysis of Machining Performance in Micro-Endmilling, Part II: Cutting Force Prediction J Manuf Sci Eng-Trans ASME 2004; 126(4),: 695-705. 
34. Dewhurst P and Collins IF. A Matrix Technique constructing Slip-Line Field Solutions to a Class of Plane Strain Plasticity Problems Int J Numer Methods Eng 1973; 7(3): 357-378.

35. Altintas Y and Jin X. Mechanics of micro-milling with round edge tools. CIRP Annals-Manufacturing Technology 2011; 60(1): 77-80

36. Annoni A, Biella G, Mayor JR, et al. Experimental verification of a slip-line field model of orthogonal cutting in the microscale. In: ICOMM $2013-8^{\text {th }}$ International Conference on MicroManufacturing, Victoria, Canada, 25 - 28 March 2013, paper n 104, pp. 491-498.

37. Vollertsen F, Biermann D, Hansen HN, et al. Size effects in manufacturing of metallic components CIRP Ann-Manuf Technol 2009; 58: 566-587. 